\title{
ON COMPOSITIONS OF DERIVATIONS OF PRIME RINGS
}

\author{
CHEN-LIAN CHUANG \\ (Communicated by Donald S. Passman)
}

\begin{abstract}
Let $R$ be a prime ring and $\varphi\left(x_{i}\right)$ be a differential polynomial of $R$. It is shown that if $\varphi\left(x_{i}\right)=0$ holds on a nonzero two-sided ideal of $R$, then $\varphi\left(x_{i}\right)=0$ holds on $R_{F}$, the left Martindale quotient ring of $R$. Using this together with Kharchenko's theorem on differential identities, we settle three problems raised by Krempa and Matczuk in the positive.
\end{abstract}

\section{INTRODUCTION}

In [4], Krempa and Matczuk raised the following problems:

Problem 1. Let $L$ be a field, $\operatorname{char} L=p<\infty$, and let $\delta_{1}, \ldots, \delta_{p}$ be such derivations of $L$ that $\varphi(x)=x^{\delta_{1}, \ldots, \delta_{p}}$ defines a derivation. Whether there exists $l \in L$ such that $\varphi(x)=l x^{\left(d^{p}\right)}$ for some derivation $d$ of $L$.

In the following, let $\delta_{1}, \ldots, \delta_{n}$ be derivations of a prime ring, $\operatorname{char} R>n$, and let $I$ be a nonzero ideal of $R$.

Problem 2. Whether $x^{\delta_{1}, \ldots, \delta_{n}}=c x$ for all $x \in I$, where $c$ is a fixed element in the extended centroid of $R$, implies $c=0$.

Problem 3. Whether $x^{\delta_{1}, \ldots, \delta_{n}}=0$ for all $x \in I$ implies $x^{\delta_{1}, \ldots, \delta_{n}}=0$ for all $x \in R$.

Our aim here is to show that all the three problems follow easily from Kharchenko's powerful theorem on differential identities.

\section{Preliminary}

Here we recall Kharchenko's theorem on differential identities and meanwhile explain our notation (adopted from [2] and [3]).

Throughout the paper, $R$ is a prime ring and $F$ is the filter of all nonzero two-sided ideals of $R$. Let $R_{F}$ and $Q$ stand for, respectively, the left Martindale quotient ring of $R$. (See p. 156 of [2] for the definitions.) The center of $R_{F}$, denoted by $C$, coincides with the center of $Q$ and is called the extended

Received by the editors September 2, 1987 and, in revised form, May 22, 1989.

1980 Mathematics Subject Classification (1985 Revision). Primary 16A72.

Key words and phrases. Prime rings, derivations, differential identities. 
centroid of $R$. A derivation $\delta$ of the ring $R$ is a map from $R$ into $R$ satisfying $(x+y)^{\delta}=x^{\delta}+y^{\delta}$ and $(x y)^{\delta}=x^{\delta} y+x y^{\delta}$ for all $x, y \in R$. Any derivation $\delta$ defined on $R$ can be uniquely extended to a derivation of $R_{F}$, which will also be denoted by $\delta$. Thus all derivations of $R$ here are implicitly assumed to be defined on the whole $R_{F}$. For a derivation $\delta$ of $R_{F}$ and for $\alpha \in C$, we define $\delta \alpha$ by $x^{\delta \alpha}=\alpha x^{\delta}$ for all $x \in R_{F}$. So $\delta \alpha$ is also a derivation of $R_{F}$. In this manner, the set of all derivations of $R_{F}$ forms a right $C$-vector space.

A derivation $\delta$ is said to be (Q-)inner if there exists $a \in Q$ such that $x^{\delta}=[x, a]=x a-a x$ for all $x \in R_{F}$.

Let $D_{\text {int }}$ be the set of all inner derivations of $R_{F} . D_{\text {int }}$ is obviously a $C$ subspace of the space of all derivations of $R_{F}$. Let $\operatorname{Der}(R)$ be the set of all derivations of $R$ and let $D$ be the $C$-subspace spanned by $\operatorname{Der}(R)$, that is, $D=\operatorname{Der}(R) \cdot C$. The $C$-subspace spanned by all derivations of $R$ and all $\left(Q\right.$-)inner derivations is obviously equal to $D_{\text {int }}+D$.

We choose a basis $M_{0}$ for $D_{\text {int }}$ and augment it to a basis $M$ of $D_{\text {int }}+D$. We also fix a total order $>$ in the set $M$ such that $\mu_{0}>\mu$ for $\mu_{0} \in M_{0}$ and $\mu \in M \backslash M_{0}$. We then extend this order to the set of all derivation words in $M$ by assuming that a longer word is greater than a shorter one and that words of the same length are ordered lexicographically. By a regular word in $M$, we mean a word of the form $\Delta=\delta_{1}^{s_{1}} \delta_{2}^{s_{2}} \cdots \delta_{m}^{s_{m}}$ such that (1) $\delta_{i} \in M \backslash M_{0}$ for $i=1, \ldots, m,(2) \delta_{1}<\delta_{2}<\cdots<\delta_{m}$, and (3) $s_{i}<p$ for $i=1, \cdots, m$, if char $R=p>0$.

Remark 1. Since $\operatorname{Der}(R)$ spans $D$ as a $C$-subspace, elements of $M \backslash M_{0}$ can be chosen from $\operatorname{Der}(R)$. In the proof of the crucial Lemma 2 of [2] (pp. 158-160), derivations in $M \backslash M_{0}$ are implicitly assumed to be in $\operatorname{Der}(R)$ and this can be done as is said above. However, as is explained in the remark on p. 74 of [3], all we need is that for each derivation $\delta \in M \backslash M_{0}$, there exists a nonzero ideal $I$ of $R$ such that $I^{\delta} \subseteq R$. Every $(Q$-)inner derivation satisfies this property by the very definition of $Q$, and every derivation of $D$ also satisfies this property, since so does every derivation in $\operatorname{Der}(R)$. So this property is obviously enjoyed by all derivations of $D_{\text {int }}+D$. For this reason, we will not put any restriction on $M$.

By a differential polynomial, we mean a generalized polynomial with coeffcients in $R_{F}$ and involving noncommuting indeterminates which are acted by compositions of derivations of $R$. For a differential polynomial $\varphi, \varphi=0$ is said to be a differential identity for $R$ if $\varphi$ assumes the constant value 0 for any assignment of values from $R$ to its indeterminates.

By means of identities 1-6, [3, p. 58], any differential polynomial $\varphi\left(x_{i}\right)$ can be transformed into the form $\psi\left(x_{i}^{\Delta_{j}}\right)$, where (1) $\psi\left(z_{i j}\right)$ is a generalized polynomial in distinct indeterminates $z_{i j}$, and where (2) $\Delta_{j}$ are regular words in 
M. $\psi\left(x_{i}^{\Delta_{j}}\right)$ is called the reduced expression of $\varphi\left(x_{i}\right)$. Kharchenko's theorem on differential identities [3, p. 65] asserts that if $\psi\left(x_{i}^{\Delta_{j}}\right)=0$ is a differential identity of $R$, where $\Delta_{j}$ are distinct regular derivation words in $M$ and where $\psi\left(z_{i j}\right)$ is a generalized polynomial, then $\psi\left(z_{i j}\right)=0$ is a generalized polynomial identity of $R$. The remark on page 74 of [3] says that the same conclusion still holds if $\varphi\left(x_{i}\right)=0$ is only assumed to hold on a nonzero ideal of $R$ instead of the whole $R$.

Remark 2. In Kharchenko's theorem quoted above, as long as two regular derivation words $\Delta, \Delta^{\prime}$ in $M$ are distinct as words, $x^{\Delta}$ and $x^{\Delta^{\prime}}$ must be substituted by distinct indeterminates, no matter whether $\Delta, \Delta^{\prime}$ are the same as maps on $R$ or not, that is, no matter whether $x^{\Delta}=x^{\Delta^{\prime}}$ holds for all $x \in R$ or not. As a consequence, two distinct regular derivation words $\Delta, \Delta^{\prime}$ can never be the same as maps on $R$ (or on a nonzero ideal of $R$ ): For if $x^{\Delta}=x^{\Delta^{\prime}}$ holds for all $x \in R$, then, by Kharchenko's theorem, the identity $y=z$, where $y, z$ are distinct indeterminates, would hold on $R$, an obvious contradiction. As an immediate corollary, if $\delta \in M \backslash M_{0}$, then $\delta^{t} \quad(1<t<\operatorname{char} R)$ can never be equal to any $\mu \in M \backslash M_{0}$ as maps, since, as regular words, $\delta^{t}$ and $\mu$ are of length $t \quad(t>1)$ and of length one, respectively, and hence must be distinct as words. Actually, if $\delta \in M \backslash M_{0}$, then $\delta^{t}(1<t<p)$ cannot be a derivation in $D_{\text {int }}+D$ (as maps): Indeed, if $\delta^{t}$ is a derivation in $D_{\text {int }}+D$, then $\delta^{t}$ must be a $C$-linear combination of derivations in $M$ (as maps). So there exist $\alpha_{i} \in C$, $\mu_{i} \in M \backslash M_{0}$ and $b \in Q$ such that $x^{\delta^{t}}=\sum_{i} \alpha_{i} x^{\mu_{i}}+[b, x]$ holds for all $x \in R$. By Kharchenko's theorem, the identity $y=\sum \alpha_{i} z_{i}+[b, x]$, where $y, z_{i}, x$ are distinct indeterminates, holds on $R$. This is absurd. These comments will help clarify parts of the argument (Cases 1 and 2) in the proof of Theorem 2.

\section{Main Results}

The following theorem is simply a combination of a remark in [3] and a theorem in [1], and can be viewed as a generalization of both.

Theorem 1. Let $R$ be a prime ring and let $R_{F}$ be its left Martindale quotient ring. Assume that $\varphi\left(x_{i}\right)$ is a differential polynomial involving only derivations of $R$, in noncommuting indeterminates $x_{i}$ and with coefficients in $R_{F}$. If $\varphi\left(x_{i}\right)$ vanishes on a nonzero ideal of $R$, then $\varphi\left(x_{i}\right)$ vanishes on $R_{F}$.

Proof. Using the basic identities 1-6 [3, p. 58] $\varphi\left(x_{i}\right)$ can be transformed into the reduced expression $\psi\left(x_{i}^{\Delta_{j}}\right)$, where $\Delta_{j}$ are regular derivation words and where $\psi\left(z_{i j}\right)$ is an ordinary generalized polynomial. By the remark on page 74 of [3], $\psi\left(z_{i j}\right)=0$ is a generalized polynomial identity of $R$. By Theorem $2[1], \psi\left(z_{i j}\right)=0$ is also a generalized polynomial identity of $R_{F}$. Thus $\psi\left(x_{i}^{\Delta j}\right)=0$ also holds on $R_{F}$, when the derivations are extended to be defined on $R_{F}$. Since the basic identities 1-6 [3, p. 58] also hold for the 
derivations thus extended, $\psi\left(x_{i}^{\Delta_{j}}\right)=0$ can be transformed back to $\varphi\left(x_{i}\right)=0$. So $\varphi\left(x_{i}\right)=0$ holds on $R$ as desired.

Actually, Theorem 1 is more or less implicitly implied in [3]. If we refer to the proof of [3] instead of theorems only, a more direct proof of Theorem 1 can be given.

Obviously, Problem 3 is only a very special instance of the above theorem. Now we prove Problem 2 (without the assumption that char $R>n$ ).

Proof of Problem 2. Suppose that $\delta_{1}, \ldots, \delta_{n}$ are derivations of a prime ring $R$ such that $x^{\delta_{1} \cdots \delta_{n}}=c x$ for all $x \in I$, where $I$ is a nonzero ideal of $R$ and $c$ is a fixed element of $C$. The differential identity $x^{\delta_{1} \cdots \delta_{n}}=c x$ holds on $I$ and hence must also hold on $R_{F}$ by Theorem 1 . Since $R_{F}$ has the identity 1 , we have $c=c 1=(1)^{\delta_{1} \cdots \delta_{n}}=0$, as desired.

Remark 3. (1) The above proof also holds for a $C$-linear combination of compositions of derivations. For example, let $\delta_{1}, \ldots, \delta_{n}, d_{1}, \ldots, d_{m}, \mu_{1}, \ldots, \mu_{l}$, $\ldots$ be derivations of $R$ and let $\alpha, \beta, \gamma, \ldots \in C$. If $\alpha x^{\delta_{1} \cdots \delta_{n}}+\beta x^{d_{1} \cdots d_{m}}+$ $\gamma x^{\mu_{1} \cdots \mu_{l}}+\cdots=c x$ for all $x \in I$ and for some fixed $c \in C$, then $c=0$. (2) In the assumption, $c$ can also be assumed to be dependent on $x$, that is, $c=c(x)$. An easy argument shows that $c(x)$ is a constant, unless $R$ is commutative.

For $a_{1}, \ldots, a_{m} \in R$, let $\left[x, a_{1}, \ldots, a_{m}\right]$ denote $\left[\left[\cdot\left[\left[x, a_{1}\right], a_{2}\right], \ldots\right], a_{m}\right]$. The proof of the following theorem, though a little bit lengthy, is still a straight forward application of Kharchenko's theorem.

Theorem 2. Let $R$ be a prime ring of characteristic $p>0$. Suppose that $\delta_{1}, \ldots, \delta_{p}$ are derivations of $R$ such that $\varphi(x)=x^{\delta_{1} \cdots \delta_{p}}$ defines a derivation on $R$. Then (1) if one of $\delta_{1}, \ldots, \delta_{p}$ is inner, then $\varphi(x)$ is also inner, (2) if all $\delta_{1}, \ldots, \delta_{p}$ are outer, then there exists $0 \neq \alpha \in C$ such that $\varphi(x)=\alpha x^{\left(\delta_{1}^{p}\right)}$.

Proof. Let $\delta$ be any derivation on $R$ and let $a_{1}, \ldots, a_{m} \in R_{F} \quad(m \geq 1)$. Observe that the identity

$$
\left[x, a_{1}, \ldots, a_{m}\right]^{\delta}=\left[x^{\delta}, a_{1}, \ldots, a_{m}\right]+\left[x, a_{1}^{\delta}, \ldots, a_{m}\right]+\cdots+\left[x, a_{1}, \ldots, a_{m}^{\delta}\right]
$$

holds for all $x \in R$. Using this identity together with the basic identities 1-6 [3, p. 58], the reduced expression $\psi(x)$ of $\varphi(x)=x^{\delta_{1} \cdots \delta_{p}}$ can be written as a sum of the terms of the form $\alpha x^{\Delta}$ or $\left[x^{\Delta}, a_{1}, \ldots, a_{m}\right](m \geq 1)$, where $\Delta$ is a regular derivation word, $\alpha \in C$ and $a_{1}, \ldots, a_{m} \in R_{F}$.

Since $\varphi(x)$ defines a derivation on $R$, there exist $\mu_{j} \in M \backslash M_{0}, \beta_{j} \in C$ and $b \in Q$ such that $\varphi(x)=\sum_{j} \beta_{j} x^{\mu_{j}}+[x, b]$. Hence

$$
\psi(x)=\sum_{j} \beta_{j} x^{\mu_{j}}+[x, b]
$$

gives a reduced linear differential identity, to which we are going to apply Theorem $2[3$, p. 65]. 
First, assume that one of $\delta_{i}(i=1, \ldots, p)$ is inner. Then $\psi(x)$ involves only terms of the form $\left[x^{\Delta}, a_{1}, \ldots, a_{m}\right](m \geq 1)$, where $\Delta$ are regular derivation words. Let

$$
\sum_{s}\left[x^{\mu_{j}}, a_{1}^{(s)}, a_{2}^{(s)}, \ldots, a_{m(s)}^{(s)}\right], \quad(m(s) \geq 1)
$$

denote the sum of all terms in $\psi(x)$ which involve the single derivation word $\mu_{j}$. (Set $s=0$, if no such terms exist.) By Theorem 2 [3],

$$
\sum_{s}\left[z, a_{1}^{(s)}, \ldots, a_{m(s)}^{(s)}\right]=\beta_{j} z
$$

is a GPI of $R$ and hence a GPI of $R_{F}$ by Theorem 1 above or Theorem 2 [1]. Set $z=1$. Then $\beta_{j}=\beta_{j} 1=\sum_{s}\left[1, a_{1}^{(s)}, \ldots, a_{m(s)}^{(s)}\right]=0 \quad(m(s) \geq 1)$. So $\beta_{j}=0$ for each $\mu_{j}$. Thus $\varphi(x)=[x, b]$ is inner as desired.

So, from now on, we assume that all $\delta_{i}(i=1, \ldots, p)$ are outer. Without loss of generality, we may assume that $\delta_{1}$ is the minimal element in $M$ with respect to $<$.

Suppose that one of $\delta_{2}, \ldots, \delta_{p}$ is $C$-independent of $\delta_{1}$ modulo $D_{\text {int }}$. Let $s>1$ be the minimal integer $\leq p$ such that $\delta_{s}$ is independent of $\delta_{1}$ modulo $D_{\text {int }}$. Without loss of generality, we may also assume that $\delta_{s}$ is the next minimal element of $M$. Then the leading word $\Delta$ of $\psi(x)$ must be of the form $\Delta=$ $\delta_{1}^{t_{1}} \delta_{s}^{t_{2}} \cdots\left(t_{1} \geq 1, t_{2} \geq 1\right)$. Since the characteristic of $R$ is $p$ and since all $\delta_{i}$ are outer, the only term of $\psi(x)$ which involves $x^{\Delta}$ is of the form $\alpha x^{\Delta}$, where $\alpha$ is a nonzero element of the extended centroid $C$. Theorem 2 [3] says that $\alpha z=0$ is a GPI of $R$. This is obviously a contradiction.

So we must assume that each of $\delta_{2}, \ldots, \delta_{p}$ is $C$-dependent on $\delta_{1}$ modulo $D_{\text {int }}$. For brevity, set $\delta=\delta_{1}$. Using the basic identities 1-6 [3, p. 58], $\varphi(x)$ can be written in the form $\varphi(x)=\alpha x^{\left(\delta^{p}\right)}+\varphi^{\prime}(x)$, where $0 \neq \alpha \in C$ and where $\varphi^{\prime}(x)$ involves only regular derivation words $\delta, \delta^{2}, \ldots, \delta^{p-1}$. By means of the identity mentioned at the beginning of the proof, $\varphi^{\prime}(x)$ can be written as a sum of terms of the form $\left[x^{\left(\delta^{t}\right)}, a_{1}, \ldots, a_{m}\right],(1 \leq t \leq p-1, m \geq 1)$. It is important to observe that $\varphi^{\prime}(x)$ contains no terms involving $x$.

Case 1. $\delta$ and $\delta^{p}$ are $C$-dependent modulo $D_{\text {int }}$ : Let us say $x^{\delta^{p}}=\gamma x^{\delta}+[x, c]$, where $\gamma \in C$ and $c \in Q$. The following facts are obvious: (1) the regular derivation words occurring in $\psi$ are $\varnothing$ (empty), $\delta, \delta^{2}, \ldots, \delta^{p-1} ;(2) \alpha[x, c]$ is the only term of $\psi(x)$ which involves $x$.

Now, we apply Theorem 2 [3]: By (2), $\alpha[x, c]=[x, b]$ for all $x \in R$. By (1), $\beta_{j}=0$ for each $\mu_{j} \neq \delta$. (Note that $\delta^{t} \quad(1<t<p)$ is distinct from $\mu_{j}$ as regular derivation words. See Remark 2.) For $\mu_{j}=\delta$, let $\sum_{s}\left[x^{\delta}, a_{1}^{(s)}, \ldots, a_{m(s)}^{(s)}\right]$ be the sum of all those terms in $\varphi^{\prime}(x)$ which involve $x^{\delta}$. By Theorem 2 [3],

$$
\gamma z+\sum_{s}\left[z, a_{1}^{(s)}, \ldots, a_{m(s)}^{(s)}\right]=\beta_{j} z
$$


is a GPI of $R$ and hence a GPI of $R_{F}$ by Theorem 1. Hence

$$
\beta_{j}=\beta_{j} 1=\alpha \gamma 1+\sum_{s}\left[1, a_{1}^{(s)}, \ldots, a_{m(s)}^{(s)}\right]=\alpha \gamma .
$$

So $\varphi(x)=\beta_{j} x^{\delta}+[x, b]=\alpha \gamma x^{\delta}+\alpha[x, c]=\alpha x^{\delta^{p}}$, as desired.

Case 2. $\delta$ and $\delta^{p}$ are $C$-independent modulo $D_{\text {int }}$ : Without loss of generality, we may also assume that $\delta^{p} \in M$. Hence $\delta^{p}$, regarded as a regular word, is of length one. Thus the only regular derivation words occurring in $\psi(x)$ are $\delta$, $\delta^{2}, \ldots, \delta^{p}$ (and no empty word $\varnothing$ ).

Now, we apply Theorem 2 [3]: For $\mu_{j} \neq \delta, \delta^{p}$, (as derivation words), we have $\beta_{j}=0$ as before. (See Remark 2.) For $\mu_{j}=\delta$, let $\sum_{s}\left[x^{\delta}, a_{1}^{(s)}\right.$, $\left.\ldots, a_{m(s)}^{(s)}\right]$ be the sum of those terms in $\varphi^{\prime}(x)$ which involve $x^{\delta}$. Then, by Theorem 2 [3],

$$
\sum_{s}\left[z, a_{1}^{(s)}, \ldots, a_{m(s)}^{(s)}\right]=\beta_{j} z
$$

is a GPI of $R$ and hence a GPI of $R_{F}$. So $\beta_{j}=\beta_{j} 1=\sum_{s}\left[1, a_{1}^{(s)}, \ldots, a_{m(s)}^{(s)}\right]=$ 0 . For $\mu_{j}=\delta^{p}, \beta_{j}=\alpha$ follows easily. Since $\psi(x)$ contains no terms involving $x,[x, b]=0$ for all $x$. Hence $\varphi(x)=\alpha x^{\delta^{p}}$, as desired.

If $R$ is a field, then $R=Q=R_{F}$ and also inner derivations of $R$ are trivial. So Problem 1 follows from Theorem 2 as a special instance.

\section{REFERENCES}

1. C.-L. Chuang, GPIs having coefficients in Utumi quotient rings, Proc. Amer. Math. Soc. 103 (1988), 723-728.

2. V. K. Kharchenko, Differential identities of prime rings, Algebra i Logika 17 (2) (1978), 220238 (English translation, 154-168).

3. __ Differential identities of semiprime rings, Algebra i Logika 18 (1) (1979), 86-119 (English translation, 58-80).

4. J. Krempa and J. Matczuk, On the composition of derivations, Rend. Circ. Mat. Palermo (2) 33 (1984), 441-455.

5. J. Lambek, Lectures in rings and modules, Chelsea Publishing Company, 1976.

Department of Mathematics, National Taiwan University, Taipei, Taiwan 10764, REPUBLIC OF CHINA 